\title{
Anxiety, Depression and Risk of Post-Traumatic Stress Disorder in Health Workers: The Relationship with Burnout during COVID-19 Pandemic in Italy
}

\author{
Lucio Ghio $^{1}$, Sara Patti ${ }^{1}$ **D , Giulia Piccinini ${ }^{1}$, Cinzia Modafferi ${ }^{2}$, Eleonora Lusetti ${ }^{1}$, Massimo Mazzella ${ }^{3}$ \\ and Massimo Del Sette ${ }^{4,5}$ \\ 1 Psychiatry Branch, Galliera Hospital, Mura delle Cappuccine, 14, 16128 Genoa, Italy; \\ lucio.ghio@asl3.liguria.it (L.G.); giulia.piccinini@asl3.liguria.it (G.P.); eleonora.lusetti@asl3.liguria.it (E.L.) \\ 2 V.I.E. Development, Innovation, Empowerment srl Spinoff, University of Genoa, Via Eugenia Ravasco 12, \\ 16128 Genoa, Italy; modafferi@vie-srl.it \\ 3 Maternal and Infant Department, Galliera Hospital, Mura delle Cappuccine, 14, 16128 Genoa, Italy; \\ massimo.mazzella@galliera.it \\ 4 Neurology Unit, Galliera Hospital, Mura delle Cappuccine, 14, 16128 Genoa, Italy; \\ massimo.del.sette@galliera.it \\ 5 San Martino Polyclinic Hospital IRCCS, Largo R. Benzi, 10, 16132 Genoa, Italy \\ * Correspondence: sara.patti@asl3.liguria.it
}

check for

updates

Citation: Ghio, L.; Patti, S.;

Piccinini, G.; Modafferi, C.; Lusetti, E.;

Mazzella, M.; Del Sette, M. Anxiety,

Depression and Risk of

Post-Traumatic Stress Disorder in Health Workers: The Relationship with Burnout during COVID-19 Pandemic in Italy. Int. J. Environ. Res. Public Health 2021, 18, 9929. https:// doi.org/10.3390/ijerph18189929

Academic Editor: Luenda E. Charles

Received: 19 August 2021

Accepted: 16 September 2021

Published: 21 September 2021

Publisher's Note: MDPI stays neutral with regard to jurisdictional claims in published maps and institutional affiliations.

Copyright: (C) 2021 by the authors Licensee MDPI, Basel, Switzerland. This article is an open access article distributed under the terms and conditions of the Creative Commons Attribution (CC BY) license (https:/ creativecommons.org/licenses/by/ $4.0 /)$.
Abstract: During the COVID-19 pandemic, healthcare workers (HW) have faced an extremely difficult work environment, with an increased workload and traumatic events. Our study aimed to investigate the impact of COVID-19 pandemic on HW's mental wellbeing. We analyzed the correlations between levels of burnout and other mental health disorders and we searched for the presence of specific risk factors of post-traumatic symptomatology related to the pandemic. A structured an on-line questionnaire and validated instruments were completed by a sample of HW from some hospitals in Genoa, Italy. Anxious, depressive, post-traumatic and other psychological symptoms were assessed and risk factors, related to the pandemic, were considered. Then, we investigated the correlation between levels of burnout and the risk of developing psychopathology. A total of $731 \mathrm{HW}$ were screened, and we found increased levels of anxiety (61\%), depression (62\%), PTSD (34\%) and high levels of burnout; especially emotional exhaustion (37\%). A statistically significant association between burnout and insomnia, depression, anxiety, and post-traumatic symptoms was demonstrated. This study indicates that during the COVID-19 pandemic, HW showed high levels of psychological distress and that burnout is an important predictor of sufferance. These findings support the idea to provide psychological and psychiatric support for HW.

Keywords: COVID-19; healthcare; healthcare workers; burnout; post-traumatic stress disorder; psychological trauma; depression; risk factors; anxiety; pandemic

\section{Introduction}

During the first Italian peak, in spring 2020, many major cities in our country faced overloaded intensive care units and emergency departments due to a high hospitalization for Sars-CoV2 disease. In Genoa, a city of almost 600,000 inhabitants, with $27.2 \%$ of the population over sixty-five years old, the daily hospitalization reached the peak of 719, $13 \%$ of those were admitted to intensive care units [1]. By 30 April 2020, Genoa faced 663 deaths because of COVID-19 disease: the death rate of the city, compared with the mean rates of the previous five years, increased by $56.1 \%$ in March 2020 and by $71.6 \%$ in April 2020 [2]. From mid-March 2020, most of the main hospitals in Genoa were converted by the regional government into "COVID-19 hospitals", where many wards were turned into areas dedicated to the treatment of COVID-19 patients. 
The COVID-19 pandemic has revealed that healthcare workers (HW) are at risk of developing psychological and psychiatric disorders due to the extreme working conditions [3], with a high rate of anxiety, depression, and insomnia among them [4-7]. These results were already suggested by literature on previous large-scale disasters, which pointed out that a high percentage of HW could experience post-traumatic stress disorder (PTSD), depression, anxiety, and substance disorders during these sanitary emergencies [8].

Literature on the COVID-19 pandemic has pointed out that there are many specific risk factors that can predict worse outcomes regarding HW's mental health status, such as the fear of being infected and/or to infect loved ones, higher exposure to death, role and less job seniority, younger age, female gender, absence of social support, and being frontline workers [2,9-14]. In addition, HW are at risk of experiencing high baseline burnout rates that were exacerbated by the increased levels of stress which occurred during thi pandemic [3,15].

Indeed, burnout and PTSD are both frequent syndromes in professionals working in emergency settings due to external stressors, such as work-related traumatic experiences [16], and they also share similar drivers, consequences, and comorbidities [17].

A previous study investigated the correlation between PTSD and burnout in an Italian sample of emergency HW before the COVID-19 pandemic and found a positive correlation between the two constructs [16].

During this pandemic, burnout was identified among HW and persistent burnout was found to be able to contribute to higher levels of anxiety, acute stress, and depersonalization symptoms [18-20].

Therefore, the aim of our study is to investigate the prevalence of PTSD, insomnia, anxiety, and depression on a population of HW highly involved in the pandemic emergency, and to evaluate if these disorders are correlated to specific COVID-19 risk factors, to better understand their specific trajectories, development, and duration in this population. Moreover, unlike most research, we decided to analyze levels of burnout in order to obtain a more comprehensive picture of HW's psychological wellbeing, trying to underline the correlation between high levels of burnout and other mental health disorders.

\section{Material and Methods}

\subsection{Study Design and Sample}

This study collects the baseline data of a larger longitudinal project on HW's mental health, which included a prompt response to their sufferance offering both psychiatric and psychological support within the hospitals.

The baseline survey was fillable online from 30 July 2020 to 30 September 2020 (about three months after the first COVID-19 peak in our country) and the second assessment was repeated after six months.

The survey was approved by the regional ethics committee (CER, Commissione etica Regionale, n.299/2020).

We analyzed baseline data collected with the survey administered to a sample of HW from Galliera, Scassi General Hospitals, and other minor hospitals of the city, that was filled online on the Lime Survey platform. All personnel working during the peak of the pandemic were asked to participate in the study, but the enrollment was voluntary, anonymous and participants could withdraw at any moment.

\subsection{Study Instrument}

The survey, designed as a structured questionnaire, was delivered on the Lime Survey platform and investigated sociodemographic characteristics, occupational variables, personal exposures to COVID-19 and validated instruments were included to assess psychological distress. The order of sociodemographic questions and of the questionnaires were randomized to minimize the bias related to the order of presentation. It required about $10-15$ min to be completed. 


\subsubsection{Depressive Symptoms}

Symptoms of depression were evaluated with the Patient Health Questionnaire- 9 (PHQ-9), which has shown a high internal consistency (Cronbach's $\alpha=0.83$ ) [21,22]. It is a self-administered questionnaire with 9 items that requires the participant to evaluate, on a 4-points Likert scale, the intensity of the symptoms in the last two weeks. There is a tenth item that evaluates the functional impairment caused by depression and explores to what extent the depression made it difficult to work and to maintain relationships.

The first 9 items contribute to the total score, which helps to stratify different severity levels of the depression (range 0-27; cut-off points of 5, 10, 15, 20 might be interpreted as representing mild, moderate, moderately severe, and severe depression).

\subsubsection{Generalized Anxiety Disorder Symptoms}

Symptoms of anxiety were assessed using the Generalized Anxiety Disorder-7 (GAD7), which has demonstrated good psychometric properties, including sensitivity and specificity for diagnostic GAD and a good reliability (Cronbach's $\alpha=0.89$ ) [23]. The instrument is self-administered and made of seven items that assess symptoms of GAD experienced over the past two weeks on a 4-points Likert scale (range 0-21; cut-off of 5, 10 and 15 might be interpreted as representing mild, moderate, and severe levels of anxiety).

\subsubsection{Insomnia Symptoms}

Symptoms of insomnia were assessed using the insomnia severity index (ISI), which has adequate internal consistency (Cronbach's $\alpha=0.74$ ) [24,25]. The ISI is a brief selfadministered scale that evaluates personal perception of insomnia on a 4-points Likert scale (range 0-28; cut-off points of 8, 15 and 22 that indicate the presence of subthreshold, moderate, and severe insomnia).

\subsubsection{Posttraumatic Stress Disorder symptoms}

To assess the perceived emotional distress that accompanies a stressful life event, we used the impact of event scale-revised (IES-R), that showed a high internal consistency (Cronbach's $\alpha=0.95$ ) [26-28]. Our participants were forced to choose the pandemic as the stressful event investigated. The scale is a self-administered instrument made up of twenty-two items divided into three scales (intrusion, avoidance and hyperarousal), evaluated by participants using a five-point Likert scale (range 0-88). In relation to PTSD, a score of 33 was considered as the cut-off point.

\subsubsection{Burnout Symptoms}

Symptoms of burnout were assessed with the Maslach burnout inventory (MBI) and cut-off criteria for Italian HW were used [29,30]. It is a self-administered scale formed by twenty-two items with seven response options on a Likert scale from zero (never) to six (every day), which creates three different components of the burnout: emotional exhaustion (EE, total range 0-54), depersonalization (DP, total range 0-30) and personal accomplishment (PA, total range 0-48). MBI score ranges were 0-14, 15-23, and 24-54 for the emotional subscale and indicate low, moderate, and high levels of EE; for depersonalization, the subscale score ranges were $0-3,4-8$ and 9-30, indicating low, moderate, and high levels of DP; finally, for the reduced personal accomplishment subscale, score ranges were 48-37, 36-30, and 29-0, indicating the presence of low, moderate and high levels of PA. MBI has shown good enough reliability for EE scale (Cronbach's $\alpha=0.88$ ) and a lower reliability for DP (Cronbach's $\alpha=0.71$ ) and PA scales (Cronbach's $\alpha=0.76$ ) [31].

\subsection{Data Analysis}

We reported the percentages of participants who rated low to high scores in psychological distress. We used a multivariate multiple regression analysis to examine the effect of demographic characteristics, personal exposure to COVID-19, and burnout on levels of depression, anxiety, insomnia, and PTSD, in order to underline the risk factors for 
developing stress response symptomatology and other forms of psychopathology. Then we divided the sample into two groups with low and medium/high levels of burnout and we used the two-sample $\mathrm{T}$ test to investigate the presence of statistically significant differences between those two groups in presenting symptoms of depression, anxiety, insomnia, or PTSD.

All statistical analyses were performed using the SPSS version 25.0 (IBM Corp., Armonk, NY, USA), and the value of statistical significance was set at $p<0.05$ (two tailed). We used a listwise deletion for the missing data.

\section{Results}

\subsection{Sample Characteristics}

A total number of 731 participants completed the screening (29.7\% of the HW employed in the hospitals enrolled in our study). Sociodemographic characteristics of the sample and mental health measures are reported in Table 1 . The mean age of our population was $48.45 \pm 9.81$. The majority of the sample $(76 \% ; n=556)$ were female and 671 respondents $(92 \%)$ reported to be directly exposed to covid-19. More than half of the population $(62 \% ; n=451)$ thought that work impacted on their psychological wellbeing and the need for psychological support was perceived by $40 \%$ of the sample $(n=296)$. A total number of 297 respondents declared experiencing sleeping $(21 \%)$ and anxiolytic medications intake $(20 \%)$ and $32 \%(n=234)$ declared a customary alcohol consumption. More than half of the sample $(62 \% ; n=453)$ presented mild $(32 \%)$ to severe $(13 \%)$ depressive symptoms, with a PHQ total mean score of 7.34, whereas 447 participants $(61 \%)$ reported having anxiety symptoms, being severe in $11 \%$ of the sample, with a GAD-7 mean score of 6.92 . Of the 731 participants, $349(48 \%)$ experienced symptoms of insomnia, with an ISI mean total score of 8.25 . Lastly, more than a third of our sample $(34 \% ; n=250)$ was experiencing post-traumatic symptomatology and nearly half of the sample was facing increased levels of burnout, especially EE was perceived by $37 \%$ of the participants $(n=270)$.

Table 1. Study sample characteristics $(n=731)$.

\begin{tabular}{|c|c|c|c|c|}
\hline Main Categories & & Variable & Frequencies & Percent \\
\hline \multirow{10}{*}{$\begin{array}{c}\text { Socio- } \\
\text { Demographic }\end{array}$} & \multirow{2}{*}{ Gender } & Female & 556 & 76 \\
\hline & & Male & 175 & 24 \\
\hline & \multirow{4}{*}{ Marital status } & Married/Common law & 483 & 66 \\
\hline & & Stable relationship (not living together) & 69 & 10 \\
\hline & & Separated/divorced & 89 & 12 \\
\hline & & Single & 90 & 12 \\
\hline & \multirow{2}{*}{ Living alone } & No & 596 & 18 \\
\hline & & Yes & 135 & 82 \\
\hline & \multirow{2}{*}{ Offspring } & No & 253 & 65 \\
\hline & & Yes & 478 & 35 \\
\hline \multirow{8}{*}{ Employment } & \multirow{4}{*}{ Job } & Medical staff & 192 & 26 \\
\hline & & Nurse & 359 & 49 \\
\hline & & Social health worker & 72 & 10 \\
\hline & & Other & 108 & 15 \\
\hline & \multirow{4}{*}{ Seniority } & Less than 10 years & 197 & 27 \\
\hline & & Between 11 and 20 years & 172 & 24 \\
\hline & & Between 21 and 30 years & 175 & 24 \\
\hline & & Over 30 years & 187 & 25 \\
\hline
\end{tabular}


Table 1. Cont.

\begin{tabular}{|c|c|c|c|c|}
\hline Main Categories & & & Frequencies & Percent \\
\hline \multirow{13}{*}{$\begin{array}{l}\text { COVID-19 specific } \\
\text { stressors }\end{array}$} & \multirow{2}{*}{$\begin{array}{l}\text { Working with COVID-19 } \\
\text { positive patients }\end{array}$} & No & 232 & 32 \\
\hline & & Yes & 499 & 68 \\
\hline & \multirow{2}{*}{$\begin{array}{l}\text { Have you changed your } \\
\text { ward? }\end{array}$} & No & 577 & 79 \\
\hline & & Yes & 154 & 21 \\
\hline & \multirow{2}{*}{$\begin{array}{l}\text { Have you been exposed to } \\
\text { covid-19? }\end{array}$} & No & 60 & 8 \\
\hline & & Yes & 671 & 92 \\
\hline & \multirow{3}{*}{ Have you been infected? } & No & 549 & 75 \\
\hline & & Yes & 114 & 16 \\
\hline & & Other & 68 & 9 \\
\hline & \multirow{2}{*}{ Beloved with COVID-19 } & No & 386 & 53 \\
\hline & & Yes & 345 & 47 \\
\hline & Beloved dead with & No & 587 & 80 \\
\hline & COVID-19 & Yes & 144 & 20 \\
\hline \multirow{10}{*}{$\begin{array}{l}\text { Other psychological } \\
\text { variables }\end{array}$} & \multirow{2}{*}{$\begin{array}{c}\text { Work impact on } \\
\text { psychological well being }\end{array}$} & No & 280 & 38 \\
\hline & & Yes & 451 & 62 \\
\hline & \multirow{2}{*}{$\begin{array}{c}\text { Need for } \\
\text { psychological support }\end{array}$} & No & 435 & 60 \\
\hline & & Yes & 296 & 40 \\
\hline & \multirow{2}{*}{ Use of sleeping medication } & No & 579 & 79 \\
\hline & & Yes & 152 & 21 \\
\hline & \multirow{2}{*}{ Use of anxiolytic medication } & No & 586 & 80 \\
\hline & & Yes & 145 & 20 \\
\hline & \multirow{2}{*}{ Use of alcohol } & No & 497 & 68 \\
\hline & & yes & 234 & 32 \\
\hline \multirow{24}{*}{$\begin{array}{l}\text { Psychological } \\
\text { Screening }\end{array}$} & \multirow{5}{*}{ PHQ9 } & None & 278 & 38 \\
\hline & & Mild & 233 & 32 \\
\hline & & Moderate & 126 & 17 \\
\hline & & Moderately severe & 71 & 10 \\
\hline & & Severe & 23 & 3 \\
\hline & \multirow{4}{*}{ GAD7 } & None & 284 & 39 \\
\hline & & Mild & 246 & 34 \\
\hline & & Moderate & 122 & 16 \\
\hline & & Severe & 79 & 11 \\
\hline & \multirow{4}{*}{ ISI } & None & 382 & 52 \\
\hline & & Minimal or none & 220 & 30 \\
\hline & & Moderate & 105 & 14 \\
\hline & & Severe & 24 & 4 \\
\hline & \multirow{2}{*}{ IESR Tot } & None & 481 & 66 \\
\hline & & Possible PTSD & 250 & 34 \\
\hline & \multirow{3}{*}{ MBI-Exhaustion } & Low & 334 & 46 \\
\hline & & Medium & 127 & 17 \\
\hline & & High & 270 & 37 \\
\hline & \multirow{3}{*}{ MBI-Depersonalization } & Low & 379 & 52 \\
\hline & & Medium & 192 & 26 \\
\hline & & High & 160 & 22 \\
\hline & \multirow{3}{*}{$\begin{array}{l}\text { MBI-Personal } \\
\text { Accomplishment }\end{array}$} & Low & 384 & 52 \\
\hline & & Medium & 202 & 28 \\
\hline & & High & 145 & 20 \\
\hline
\end{tabular}

PHQ-9 = Patient health questionnaire-9; GAD-7 = generalized anxiety disorder-7; ISI = insomnia severity index; IES-R = impact of event scale-revisedMBI = Maslach burnout inventory. 


\subsection{Identification of Post-Traumatic Symptoms and Other Psychopathology Risk Factors}

We searched if some of the considered variables had a direct influence on PTSD (as measured by IES-R), depression (as measured by PHQ9), anxiety (as measured by GAD 7) or insomnia (as measured by ISI) with multivariate multiple regression analyses (data are reported in Table 2).

Table 2. Results of Multivariate Multiple Regression.

\begin{tabular}{|c|c|c|c|c|c|c|c|c|c|c|c|c|}
\hline \multirow{2}{*}{$\begin{array}{c}\text { Psychopatology } \\
\text { Predictor }\end{array}$} & \multicolumn{3}{|c|}{ IES-R Tot } & \multicolumn{3}{|c|}{ PHQ9 } & \multicolumn{3}{|c|}{ GAD7 } & \multicolumn{3}{|c|}{ ISI } \\
\hline & $\beta(\mathrm{SE})$ & $\mathbf{t}$ & $p$-Value & $\beta(\mathrm{SE})$ & $\mathbf{t}$ & $p$-Value & $\beta(\mathrm{SE})$ & $\mathbf{t}$ & $p$-Value & $\beta(\mathrm{SE})$ & $\mathbf{t}$ & $p$-Value \\
\hline (Intercept) & $\begin{array}{l}13.98 \\
(5.18)\end{array}$ & 2.70 & $0.001 * *$ & $\begin{array}{l}4.48 \\
(1.45)\end{array}$ & 3.08 & $0.002 *$ & $\begin{array}{l}5.05 \\
(1.38)\end{array}$ & 3.67 & $<0.001^{* * *}$ & $\begin{array}{c}2.77 \\
(1.78)\end{array}$ & 1.56 & 0.119 \\
\hline Age & $\begin{array}{l}-0.04 \\
(0.09)\end{array}$ & -0.52 & 0.600 & $\begin{array}{c}0.03 \\
(0.02)\end{array}$ & 1.32 & 0.188 & $\begin{array}{l}-0.02 \\
(0.02)\end{array}$ & -1.03 & 0.305 & $\begin{array}{c}0.03 \\
(0.03)\end{array}$ & 1.17 & 0.244 \\
\hline Gender-male & $\begin{array}{l}-3.56 \\
(1.29)\end{array}$ & -2.75 & $0.006^{* *}$ & $\begin{array}{l}-0.64 \\
(0.36)\end{array}$ & -1.76 & 0.079 & $\begin{array}{l}-0.65 \\
(0.34)\end{array}$ & -1.89 & 0.058 & $\begin{array}{l}-0.10 \\
(0.44)\end{array}$ & -0.23 & 0.819 \\
\hline Job-Medical staff & $\begin{array}{c}0.59 \\
(1.69)\end{array}$ & 0.34 & 0.731 & $\begin{array}{l}-0.02 \\
(0.48)\end{array}$ & -0.05 & 0.958 & $\begin{array}{l}0.38 \\
(0.45)\end{array}$ & 0.85 & 0.397 & $\begin{array}{l}-0.43 \\
(0.58)\end{array}$ & -0.74 & 0.459 \\
\hline Job-Nurse & $\begin{array}{l}-0.32 \\
(1.55)\end{array}$ & -0.21 & 0.838 & $\begin{array}{l}-0.63 \\
(0.44)\end{array}$ & -1.45 & 0.149 & $\begin{array}{l}-0.27 \\
(0.41)\end{array}$ & -0.67 & 0.506 & $\begin{array}{l}-0.32 \\
(0.53)\end{array}$ & -0.60 & 0.548 \\
\hline $\begin{array}{c}\text { Job-Social Health } \\
\text { worker }\end{array}$ & $\begin{array}{c}0.71 \\
(2.19)\end{array}$ & 0.32 & 0.747 & $\begin{array}{l}-0.48 \\
(0.62)\end{array}$ & -0.79 & 0.431 & $\begin{array}{c}0.57 \\
(0.58)\end{array}$ & 0.98 & 0.329 & $\begin{array}{l}-1.07 \\
(0.75)\end{array}$ & -1.42 & 0.156 \\
\hline $\begin{array}{l}\text { Seniority- } \\
11-20 \text { years }\end{array}$ & $\begin{array}{l}-1.28 \\
(2.22)\end{array}$ & -0.58 & 0.565 & $\begin{array}{l}-0.87 \\
(0.62)\end{array}$ & -1.40 & 0.162 & $\begin{array}{l}-0.01 \\
(0.59)\end{array}$ & -0.02 & 0.981 & $\begin{array}{l}-0.27 \\
(0.76)\end{array}$ & -0.36 & 0.718 \\
\hline $\begin{array}{l}\text { Seniority- } \\
21-30 \text { years }\end{array}$ & $\begin{array}{l}-1.89 \\
(1.69)\end{array}$ & -1.12 & 0.263 & $\begin{array}{l}-0.62 \\
(0.47)\end{array}$ & -1.31 & 0.191 & $\begin{array}{c}0.04 \\
(0.49)\end{array}$ & 0.09 & 0.924 & $\begin{array}{l}-0.52 \\
(0.58)\end{array}$ & -0.89 & 0.369 \\
\hline $\begin{array}{l}\text { Seniority-Over } \\
30 \text { years }\end{array}$ & $\begin{array}{l}0.93 \\
(1.94)\end{array}$ & 0.48 & 0.633 & $\begin{array}{l}-1.09 \\
(0.55)\end{array}$ & -1.99 & $0.046 *$ & $\begin{array}{l}-0.36 \\
(0.51)\end{array}$ & -0.69 & 0.489 & $\begin{array}{l}-0.39 \\
(0.66)\end{array}$ & -0.59 & 0.549 \\
\hline $\begin{array}{l}\text { COVID-19 specific } \\
\text { stressors }\end{array}$ & $\begin{array}{c}0.57 \\
(1.94)\end{array}$ & 0.29 & 0.770 & $\begin{array}{l}-0.57 \\
(0.54)\end{array}$ & -1.06 & 0.289 & $\begin{array}{l}0.19 \\
(0.51)\end{array}$ & 0.39 & 0.699 & $\begin{array}{c}1.23 \\
(0.66)\end{array}$ & 1.86 & 0.064 \\
\hline Infected No & $\begin{array}{l}0.65 \\
(1.93)\end{array}$ & 0.34 & 0.736 & $\begin{array}{l}-0.45 \\
(0.54)\end{array}$ & -0.83 & 0.406 & $\begin{array}{l}-0.70 \\
(0.51)\end{array}$ & -1.38 & 0.169 & $\begin{array}{c}0.29 \\
(0.66)\end{array}$ & 0.44 & 0.663 \\
\hline Infected Yes & $\begin{array}{l}1.33 \\
(2.25)\end{array}$ & 0.59 & 0.554 & $\begin{array}{c}0.24 \\
(0.64)\end{array}$ & 0.38 & 0.705 & $\begin{array}{l}-0.41 \\
(0.59)\end{array}$ & -0.69 & 0.488 & $\begin{array}{c}0.38 \\
(0.77)\end{array}$ & 0.50 & 0.617 \\
\hline $\begin{array}{l}\text { Beloved with } \\
\text { covid-Yes }\end{array}$ & $\begin{array}{c}0.53 \\
(1.25)\end{array}$ & 0.42 & 0.674 & $\begin{array}{l}0.19 \\
(0.35)\end{array}$ & 0.56 & 0.579 & $\begin{array}{l}0.16 \\
(0.33)\end{array}$ & 0.48 & 0.633 & $\begin{array}{l}-0.11 \\
(0.43)\end{array}$ & -0.25 & 0.803 \\
\hline $\begin{array}{l}\text { Beloved dead with } \\
\text { covid_-Yes }\end{array}$ & $\begin{array}{l}1.59 \\
(1.56)\end{array}$ & 1.02 & 0.307 & $\begin{array}{l}-0.35 \\
(0.44)\end{array}$ & -0.79 & 0.431 & $\begin{array}{c}0.21 \\
(0.41)\end{array}$ & 0.52 & 0.604 & $\begin{array}{c}0.22 \\
(0.53)\end{array}$ & 0.41 & 0.681 \\
\hline $\begin{array}{l}\text { Work impact on } \\
\text { wellbeing-Yes }\end{array}$ & $\begin{array}{l}5.73 \\
(1.29)\end{array}$ & 4.45 & $<0.001 * * *$ & $\begin{array}{l}0.45 \\
(0.36)\end{array}$ & 1.26 & 0.209 & $\begin{array}{l}0.69 \\
(0.34)\end{array}$ & 2.03 & 0.043 * & $\begin{array}{c}0.46 \\
(0.44)\end{array}$ & 1.05 & 0.292 \\
\hline $\begin{array}{c}\text { Need for } \\
\text { psychological } \\
\text { support_Yes }\end{array}$ & $\begin{array}{c}7.19 \\
(1.29)\end{array}$ & 5.58 & $<0.001^{* * *}$ & $\begin{array}{c}1.91 \\
(0.36)\end{array}$ & 5.28 & $<0.001^{* * *}$ & $\begin{array}{l}1.71 \\
(0.34)\end{array}$ & 4.99 & $<0.001^{* * *}$ & $\begin{array}{c}2.11 \\
(0.44)\end{array}$ & 4.77 & $<0.001^{* * *}$ \\
\hline $\begin{array}{c}\text { Sleeping } \\
\text { medication-Yes }\end{array}$ & $\begin{array}{c}2.72 \\
(1.49)\end{array}$ & 1.84 & 0.067 & $\begin{array}{c}0.94 \\
(0.42)\end{array}$ & 2.27 & $0.024 *$ & $\begin{array}{c}0.61 \\
(0.39)\end{array}$ & 1.54 & 0.124 & $\begin{array}{c}2.71 \\
(0.51)\end{array}$ & 5.33 & $<0.001^{* * *}$ \\
\hline $\begin{array}{c}\text { Anxiolytic } \\
\text { medication-Yes }\end{array}$ & $\begin{array}{l}3.43 \\
(1.54)\end{array}$ & 2.23 & 0.026 * & $\begin{array}{l}1.86 \\
(0.43)\end{array}$ & 4.31 & $<0.001^{* * *}$ & $\begin{array}{l}1.31 \\
(0.41)\end{array}$ & 3.22 & $0.002 * *$ & $\begin{array}{c}0.77 \\
(0.53)\end{array}$ & 1.46 & 0.145 \\
\hline Alcohol-Yes & $\begin{array}{l}-0.46 \\
(1.19)\end{array}$ & -0.39 & 0.698 & $\begin{array}{l}-0.02 \\
(0.33)\end{array}$ & -0.05 & 0.963 & $\begin{array}{l}0.09 \\
(0.32)\end{array}$ & 0.31 & 0.759 & $\begin{array}{l}-0.01 \\
(0.41)\end{array}$ & 0.00 & 0.999 \\
\hline $\begin{array}{c}\text { MBI } \\
\text { Depersonalization }\end{array}$ & $\begin{array}{l}0.18 \\
(0.11)\end{array}$ & 1.66 & 0.098 & $\begin{array}{l}-0.01 \\
(0.03)\end{array}$ & -0.37 & 0.711 & $\begin{array}{l}-0.01 \\
(0.02)\end{array}$ & -0.07 & 0.947 & $\begin{array}{l}-0.07 \\
(0.04)\end{array}$ & -1.82 & 0.069 \\
\hline $\begin{array}{c}\text { MBI Personal } \\
\text { Accomplishment }\end{array}$ & $\begin{array}{l}-0.09 \\
(0.06)\end{array}$ & -1.37 & 0.172 & $\begin{array}{l}-0.06 \\
(0.01)\end{array}$ & -3.50 & $<0.001^{* * *}$ & $\begin{array}{l}-0.05 \\
(0.02)\end{array}$ & -2.79 & $0.005^{* *}$ & $\begin{array}{l}-0.07 \\
(0.02)\end{array}$ & -3.01 & $0.002^{* *}$ \\
\hline
\end{tabular}


Table 2. Cont.

\begin{tabular}{|c|c|c|c|c|c|c|c|c|c|c|c|c|}
\hline \multirow{2}{*}{$\begin{array}{c}\text { Psychopatology } \\
\text { Predictor }\end{array}$} & \multicolumn{3}{|c|}{ IES-R Tot } & \multicolumn{3}{|c|}{ PHQ9 } & \multicolumn{3}{|c|}{ GAD7 } & \multicolumn{3}{|c|}{ ISI } \\
\hline & $\beta(\mathrm{SE})$ & $\mathbf{t}$ & $p$-Value & $\beta(\mathrm{SE})$ & $t$ & $p$-Value & $\beta(\mathrm{SE})$ & $t$ & $p$-Value & $\beta(\mathrm{SE})$ & $t$ & $p$-Value \\
\hline $\begin{array}{l}\text { MBI_Emotional } \\
\text { Fatigue }\end{array}$ & $\begin{array}{c}0.45 \\
(0.05)\end{array}$ & 8.94 & $<0.001^{* * *}$ & $\begin{array}{c}0.19 \\
(0.01)\end{array}$ & 13.91 & $<0.001^{* * *}$ & $\begin{array}{c}0.18 \\
(0.01)\end{array}$ & 13.63 & $<0.001^{* * *}$ & $\begin{array}{c}0.19 \\
(0.02)\end{array}$ & 11.31 & $<0.001 * * *$ \\
\hline R 2 (adjusted R 2) & \multicolumn{3}{|c|}{$0.49(0.47)$} & \multicolumn{3}{|c|}{$0.57(0.55)$} & \multicolumn{3}{|c|}{$0.56(0.55)$} & \multicolumn{3}{|c|}{$0.46(0.44)$} \\
\hline Residual std. error & \multicolumn{3}{|c|}{13.56} & \multicolumn{3}{|c|}{3.81} & \multicolumn{3}{|c|}{3.60} & \multicolumn{3}{|c|}{4.65} \\
\hline F statistic $(\mathrm{df}=21,672)$ & \multicolumn{3}{|c|}{$30.21^{* * *}$} & \multicolumn{3}{|c|}{$41.62^{* * *}$} & \multicolumn{3}{|c|}{$40.74^{* * *}$} & \multicolumn{3}{|c|}{$27.38^{* * *}$} \\
\hline
\end{tabular}

IES-R = Impact of event scale-revised; PHQ-9 = patient health questionnaire-9; GAD-7 = generalized anxiety disorder-7; ISI = insomnia severity index; $\mathrm{SE}=$ standard error; ${ }^{* * *}=$ significant for $p<0.001,{ }^{* *}=$ significant for $p<0.01 .{ }^{*}=$ significant for $p<0.05$.

We found that male gender was a protective factor in developing PTSD, having a negative association with post-traumatic symptomatology (Std. $\beta=-0.28, p=0.006$ ); similarly, seniority over 30 years had a negative association with depressive symptoms (Std. $\beta=-0.02, p=0.046$ ). On the other hand, the need for psychological support was associated with higher levels of post-traumatic symptomatology (Std. $\beta=0.56, p<0.001$ ), depressive symptoms (Std. $\beta=0.16, p<0.001$ ), anxiety (Std. $\beta=0.13, p<0.001$ ), and insomnia (Std. $\beta=0.18, p<0.001$ ); while the perception of work impacting on wellbeing was associated with post-traumatic symptomatology (Std. $\beta=0.52, p=0.001$ ), and anxiety (Std. $\beta=0.06, p=0.043$ ).

We also found a positive association between the use of sleeping medication and depression (Std. $\beta=0.07, p=0.024$ ) and insomnia (Std. $\beta=0.20, p<0.001$ ); while the use of anxiolytic drugs was linked to post-traumatic (Std. $\beta=0.24, p=0.026$ ), depressive (Std. $\beta=0.11, p<0.001$ ), and anxiety symptoms (Std. $\beta=0.09, p=0.002$ ).

Lastly, looking at the correlation between burnout and psychopathology, we found that EE was associated with PTSD (Std. $\beta=1.18, p<0.001$ ), depression (Std. $\beta=0.47$, $p<0.001$ ), anxiety (Std. $\beta=0.48, p<0.001$ ), and insomnia (Std. $\beta=0.47, p<0.001$ ); while low scores on the PA subscale were associated with higher depressive (Std. $\beta=-0.10$, $p<0.001$ ) and anxiety symptoms (Std. $\beta=0.02, p=0.005$ ).

\subsection{Burnout Impact on Health Workers Mental Health}

In Table 3, we report descriptive statistics for the instruments used to evaluate the presence of psychopathology. We divided the sample into low and moderate-high risk of burnout reporting the mean score for each scale of psychopathology (PHQ-9, GAD7, ISI and IES-R) stratified for each burnout subscale. The last column of the table reports results of the two-sample t-test showing the risk of developing clinical symptomatology based on the levels of burnout. Results demonstrate that medium/high scores in all burnout subscales have a statistically significant association with insomnia, depression, anxiety, and post-traumatic symptoms $(p<0.001)$. EE appears to have the strongest impact on the whole range of the psychopathological presentation (Cohen's $d>0.80$ ), being a strong predictor of future symptomatology; whereas the other subscales (DP and PA) show only a moderate impact (Cohen's $d=0.50-0.79)$, with the only exception of PA having a low impact on insomnia symptomatology (Cohen's $d=0.48)$. 
Table 3. Descriptive statistics for measured variables and t-tests for burnout differences.

\begin{tabular}{|c|c|c|c|c|c|}
\hline Variable & $\begin{array}{l}\text { Mean and } \\
\text { Standard } \\
\text { Deviation }\end{array}$ & MBI & $\begin{array}{l}\text { Mean and Standard } \\
\text { Deviation (Low Risk) }\end{array}$ & $\begin{array}{l}\text { Mean and Standard Deviation } \\
\text { (Medium and High Risk) }\end{array}$ & $\begin{array}{l}t \text {-Test for Risk of Burnout } \\
\text { Differences }\end{array}$ \\
\hline \multirow{3}{*}{ PHQ9 } & \multirow{3}{*}{$7.32 \pm 5.69$} & $\mathrm{DP}$ & $5.70 \pm 4.90$ & $9.10 \pm 5.96$ & \multirow{3}{*}{$\begin{array}{c}\mathrm{t}(638.19)=-8.17, p<0.001 * * * \\
\text { Cohen's } \mathrm{d}=0.62 \\
\mathrm{t}(647.77)=-18.9, p<0.001 \\
\text { Cohen's } \mathrm{d}=1.39 \\
\mathrm{t}(606.46)=-8.67, p<0.001 \\
\text { Cohen's } \mathrm{d}=0.67\end{array}$} \\
\hline & & $\mathrm{EE}$ & $3.78 \pm 3.48$ & $10.28 \pm 5.48$ & \\
\hline & & PA & $5.61 \pm 4.66$ & $9.23 \pm 6.11$ & \\
\hline \multirow{3}{*}{ GAD7 } & \multirow{3}{*}{$6.86 \pm 5.84$} & DP & $5.35 \pm 4.74$ & $8.53 \pm 5.49$ & \multirow{3}{*}{$\begin{array}{c}\mathrm{t}(653.49)=-8.14, p<0.001 \\
\text { Cohen's } \mathrm{d}=0.62 \\
\mathrm{t}(643.54)=-18.98, p<0.001 \\
\text { Cohen's } \mathrm{d}=1.39 \\
\mathrm{t}(646.39)=-7.72, p<0.001 \\
\text { Cohen's } \mathrm{d}=0.59\end{array}$} \\
\hline & & $\mathrm{EE}$ & $3.53 \pm 3.23$ & $9.65 \pm 5.17$ & \\
\hline & & PA & $5.43 \pm 4.74$ & $8.47 \pm 5.53$ & \\
\hline \multirow{3}{*}{ ISI } & \multirow{3}{*}{$8.19 \pm 6.23$} & $\mathrm{DP}$ & $6.80 \pm 5.58$ & $9.73 \pm 6.55$ & \multirow{3}{*}{$\begin{array}{c}\mathrm{t}(649.6)=-6.31, p<0.001 * * * \\
\text { Cohen's } \mathrm{d}=0.48 \\
\mathrm{t}(672.78)=-16.44, p<0.001 * * * \\
\text { Cohen's } \mathrm{d}=1.22 \\
\mathrm{t}(661.09)=-7.39, p<0.001 \\
\text { Cohen's } \mathrm{d}=0.56\end{array}$} \\
\hline & & $\mathrm{EE}$ & $4.63 \pm 4.22$ & $11.14 \pm 6.08$ & \\
\hline & & PA & $6.59 \pm 5.71$ & $9.98 \pm 6.32$ & \\
\hline \multirow{3}{*}{ IES-R } & \multirow{3}{*}{$26.6 \pm 18.62$} & DP & $20.93 \pm 15.23$ & $32.85 \pm 19.99$ & \multirow{3}{*}{$\begin{array}{c}\mathrm{t}(612.54)=-8.77, p<0.001 * * * \\
\text { Cohen's } \mathrm{d}=0.68 \\
\mathrm{t}(676.17)=-15.72, p<0.001 \text { *** } \\
\text { Cohen's } \mathrm{d}=1.16 \\
\mathrm{t}(649.62)=-6.99, p<0.001 * * * \\
\text { Cohen's } \mathrm{d}=0.54\end{array}$} \\
\hline & & $\mathrm{EE}$ & $16.38 \pm 13.06$ & $35.14 \pm 18.28$ & \\
\hline & & PA & $22.05 \pm 16.75$ & $31.7 \pm 19.31$ & \\
\hline
\end{tabular}

MBI = Maslach burnout inventory; DP = depersonalization; $\mathrm{EE}=$ emotional exhaustion; $\mathrm{PA}=$ personal accomplishment; $\mathrm{PHQ}-9$ = patient health questionnaire-9; GAD-7 = generalized anxiety disorder-7; ISI = insomnia severity index; IES-R = event scale-revised; ${ }^{* * *}=$ significant $^{*}$ for $p<0.001$.

\section{Discussion}

The aim of our survey was to investigate the mental health wellbeing of Italian HW during the first outbreak of the COVID-19 pandemic. We analyzed the presence of depression, anxiety, insomnia, and post-traumatic symptomatology, as the literature describes in these emergency situations [32]; and we tried to find major and specific risk factors implied in the development of psychopathology.

We have also looked to see if burnout, already very common among HW, had a specific role in promoting or worsening the symptomatology.

As other studies show, we found high levels of mental distress, especially elevated levels of anxiety and depression in more than half of our participants $(62 \%$ and $61 \%$ respectively) [33]. Our results show some slight differences from international studies, with a higher rate of depression compared with other research and, when compared to a recent review reporting PTSD prevalence among HW during COVID-19 pandemic, lower post-traumatic symptomatology $[34,35]$. However, other findings coincide with our observations, such as a study from Bassi and colleagues that found a prevalence of PTSD of $39.8 \%$ on HW in Lombardy [36].

We also observed that $62 \%$ of participants believed that work had an important impact on their psychological wellbeing and even if we did not find a strong correlation between COVID-19 specific factors and psychopathology, we reported that, in our sample, the feeling of work impacting on one's own psychological wellbeing was associated with posttraumatic symptomatology $(p<0.001$, Table 2$)$; as we can see in other studies exploring the relationship between the pandemic and work impact [37].

Almost $40 \%$ of HW felt the need for psychological support; previous literature highlighted how the perception of needing psychological support was strongly associated with higher levels of psychological distress and also in our study it was a strong predictor for all the psychopathology examined (Table 2) [38]. 
As well as in other studies, male gender and work seniority turned out to be protective factors against the development of depression, but not other mental health disorders [39].

Regarding burnout, in our sample we found a moderate/severe presence of burnout, with high scores in each subscale, above all in EE (Table 1). Our findings agree with other observations [40,41]; in particular a recent review from Sanghera and colleagues found a prevalence of burnout between 3.1\% to $43.0 \%$ among HW during COVID-19 and Barello and colleagues found high levels of EE in $37 \%$ of the participants, from an Italian sample of HW directly employed in COVID-19 units [42,43].

To our knowledge, this is the first study investigating the correlation between levels of burnout and the risk for other mental health disorders during the COVID-19 pandemic. We found burnout was an important predictor of psychological sufferance during COVID19 outbreak among healthcare professionals. Particularly, we found a linear association between levels of EE and all the psychopathology investigated (depression, anxiety, insomnia, and post-traumatic symptoms), whereas PA was associated with higher levels of anxiety and depression. Moreover, when our sample was divided by the presence of moderate/severe burnout, we could underline a strong association between all the subscales of burnout and other psychopathology, with EE was confirmed as the most important predictor of psychological sufferance among HW.

Our findings fit with the theoretical model of burnout, where among the three dimensions of burnout, EE appears to be the most predictive factor for stress-related symptomatology, both somatic (headaches, somatic fatigue, gastrointestinal disorders) and psychological [44]. In fact, recent and previous studies on HW health during pandemic outbreaks (COVID-19, severe acute respiratory syndrome (SARS), Middle East respiratory syndrome (MERS), and H1N1 influenza) demonstrate that burnout is associated with increased risk of manifesting somatic and psychic symptoms [43,45] and it has a direct impact on stress-related symptomatology, anxiety, mood disorders, substance abuse, suicides and also on professional performance, early retirements or unexpected resignations [46].

Between all of burnout's dimension, EE is the first to develop and this could explain its higher prevalence, whereas detachment and cynicism, correlated to other subscales, often appear later [45].

These results require special attention, as previous studies showed that EE is frequently associated with suboptimal patients' care and professional inefficiencies along with a longlasting effect on health professionals' health status [47,48].

From the perspective of clinical implication, we aimed to detect psychological distress among HW in order to offer stepped care support to prevent the development of severe psychopathology and to maintain an optimal level of patients' care during the pandemic. Even outside the COVID-19 emergency, taking care of HW's levels of burnout could lead to better job performances. For these reasons, at the end of the survey we gave information about mental health support and care that were given within the hospitals enrolled in the study, in order to decrease their employees' levels of sufferance.

Our survey was conducted almost twelve-to-sixteen weeks after the first peak of the pandemic in Italy; this could be seen as a bias, taking into account that other factors not specifically inquired (such as safety precautions, the presence of protective equipment that lacked during the first weeks of the pandemic, the quarantine, etc.) could have affected the presentation of symptomatology, and the delay in the data collection could have missed the acute stress of the first phase of the pandemic. Nevertheless, our results are consistent with studies that highlight that during the initial phase of the outbreak, the symptoms of anxiety and acute stress usually prevailed, whether during the "repair" phase, when the infection starts to be under control, usually symptoms of depression and avoidance arise [49].

Another limit to our study is that, even if the survey was submitted to the all the HW employed in COVID-19 hospitals and the majority of them (68\%) had worked with COVID-19 patients, we assumed that a higher percentage of acute stress and post-traumatic stress symptomatology could be found more likely in professionals working in specific wards (as intensive and sub-intensive care units or emergency departments). Regardless, 
we decided not to investigate the specific workplace in order to increase the perception of privacy and confidentiality involved in the survey compilation.

Moreover, the use of self-report instruments is a limit to our research, which allowed us to ensure privacy and to reach a bigger number of HW through the platform, but we were not able to compare the data with previous information on participants' health records to see if there were professionals with a higher risk to develop mental health illnesses.

Furthermore, we could not collect the baseline levels of burnout, even if we run previous surveys regarding levels of burnout in HW, but only in specific professional categories. We need to compare the basal levels of burnout with those that arise in emergency situations, in order to follow changes and the development of the severity of the presentation and their association with symptomatology over time. Additionally, further longitudinal research should enquire about the role of burnout in developing psychiatric symptomatology among HW.

Lastly, these findings need to be replicated in larger samples, in fact only a third of HW working in the hospitals enrolled in our study responded to our survey, and it should be compared to symptomatology in the general population.

All the associations studied are in the expected direction: people who feel their job have an impact on their wellbeing and they are more willing to seek psychological support, show higher levels of burnout with a more cynical and detached attitude towards patients and colleagues, and are likely to present depression, anxiety, and post-traumatic symptoms, and insomnia. These findings support the usefulness and suitability of our project, integrated with our survey study, to offer stable, worker-friendly, fitting with the work schedule, psychological and psychiatric support in order to decrease the burden and the sufferance that this pandemic has inflicted on HW [50-54]. It is important to embed in the workplace targeted and specific prevention strategies, especially in healthcare settings, in order to decrease levels of burnout, to improve mental health wellbeing and resilience, and to ensure a safe and supportive environment that leads to better psychological and physical wellbeing.

\section{Conclusions}

The rapid spread of the COVID-19 pandemic had shown how HW could present high levels of psychological distress and burnout, and the latter was found to be an important predictor of sufferance.

This pandemic has enlightened the need for stable psychological support for HW, not only during the peak of the pandemic, when the work overload and other factors that impact on HW mental health are more defined, but also in everyday situations that already challenge HW regarding their mental and physical wellbeing, as literature on burn-out indicates.

Author Contributions: L.G., M.M. and M.D.S., conceptualization, supervision, validation; E.L., methodology, data curation; C.M., methodology, formal analysis; G.P., writing-original draft preparation; S.P., writing—original draft preparation, and editing. All authors have read and agreed to the published version of the manuscript.

Funding: This research received no external funding.

Institutional Review Board Statement: The study was conducted according to the guidelines of the Declaration of Helsinki, and the permission was obtained by the Ethic Commission of our Hospital-Galliera Hospital, Genoa (Italy).

Informed Consent Statement: Written informed consent was obtained from the healthcare workers to publish this paper.

Data Availability Statement: The data presented in this study are available on request from the corresponding author. The data are not publicly available due to HW' privacy.

Acknowledgments: The authors would like to thank all the health workers who enrolled and were part of this study. 
Conflicts of Interest: The authors declare no conflict of interest.

\section{References}

1. Ministero Della Salute [Ministry of Health]. COVID-19-Situazione in Italia [COVID-19-The Situation in Italy]. 2020. Available online: http:/ / www.salute.gov.it/portale/nuovocoronavirus/dettaglioContenutiNuovoCoronavirus.jsp?area=nuovoCoronavirus\& $\mathrm{id}=5351 \&$ lingua $=$ italiano\&menu=vuoto (accessed on 10 June 2020).

2. Istat, Impatto Dell'Epidemia Covid-19 Sulla Mortalità Totale Della Popolazione Residente Primo Quadrimestre 2020, Giugno. 2020. Available online: https://www.epicentro.iss.it/coronavirus/pdf/Rapp_Istat_Iss_3Giugno.pdf (accessed on 10 June 2020).

3. Restauri, N.; Sheridan, A.D. Burnout and Posttraumatic Stress Disorder in the Coronavirus Disease 2019 (COVID-19) Pandemic: Intersection, Impact, and Interventions. J. Am. Coll. Radiol. 2020, 17, 921-926. [CrossRef] [PubMed]

4. $\quad$ Lee, A.M.; Wong, J.G.; McAlonan, G.M.; Cheung, V.; Cheung, C.; Sham, P.C.; Chu, C.-M.; Wong, P.-C.; Tsang, K.W.; Chua, E.S. Stress and Psychological Distress among SARS Survivors 1 Year after the Outbreak. Can. J. Psychiatry 2007, 52, 233-240. [CrossRef]

5. Maunder, R.; Hunter, J.; Vincent, L.; Bennett, J.; Peladeau, N.; Leszcz, M.; Sadavoy, J.; Verhaeghe, L.M.; Steinberg, R.; Mazzulli, T. The immediate psychological and occupational impact of the 2003 SARS outbreak in a teaching hospital. CMAJ 2003, 168, 1245-1251. [PubMed]

6. Ricci Cabello, I.; Meneses-Echavez, J.F.; Serrano-Ripoll, M.J.; Fraile-Navarro, D.; Fiolde Roque, M.A.; Pastor Moreno, G.; Castro, A.; Ruiz-Pérez, I.; Zamanillo Campos, R.; Gonçalves-Bradley, D. Impact of viral epidemic outbreaks on mental health of healthcare workers: A rapid systematic review. medRxiv 2020. [CrossRef]

7. Chew, N.W.; Lee, G.K.; Tan, B.Y.; Jing, M.; Goh, Y.; Ngiam, N.J.; Yeo, L.; Ahmad, A.; Khan, F.A.; Shanmugam, G.N.; et al. A multinational, multicentre study on the psychological outcomes and associated physical symptoms amongst healthcare workers during COVID-19 outbreak. Brain Behav. Immun. 2020, 88, 559-565. [CrossRef]

8. Neria, Y.; Nandi, A.; Galea, S. Post-traumatic stress disorder following disasters: A systematic review. Psychol. Med. 2008, 38 , 467-480. [CrossRef]

9. Kang, L.; Ma, S.; Chen, M.; Yang, J.; Wang, Y.; Li, R.; Yao, L.; Bai, H.; Cai, Z.; Yang, B.X.; et al. Impact on mental health and perceptions of psychological care among medical and nursing staff in Wuhan during the 2019 novel coronavirus disease outbreak: A cross-sectional study. Brain Behav. Immun. 2020, 87, 11-17. [CrossRef] [PubMed]

10. Lai, J.; Ma, S.; Wang, Y.; Cai, Z.; Hu, J.; Wei, N.; Wu, J.; Du, H.; Chen, T.; Li, R.; et al. Factors Associated with Mental Health Outcomes among Health Care Workers Exposed to Coronavirus Disease 2019. JAMA Netw. Open 2020, 3, e203976. [CrossRef]

11. Mrklas, K.; Shalaby, R.; Hrabok, M.; Gusnowski, A.; Vuong, W.; Surood, S.; Urichuk, L.; Li, D.; Li, X.-M.; Greenshaw, A.J.; et al. Prevalence of Perceived Stress, Anxiety, Depression, and Obsessive-Compulsive Symptoms in Health Care Workers and Other Workers in Alberta during the COVID-19 Pandemic: Cross-Sectional Survey. JMIR Ment. Health 2020, 7, e22408. Available online: https:/ / preprints.jmir.org/preprint/22408 (accessed on 24 March 2021). [CrossRef] [PubMed]

12. Spoorthy, M.S.; Pratapa, S.K.; Mahant, S. Mental health problems faced by healthcare workers due to the COVID-19 pandemic-A review. Asian J. Psychiatry 2020, 51, 102119. [CrossRef] [PubMed]

13. Wu, P.; Fang, Y.; Guan, Z.; Fan, B.; Kong, J.; Yao, Z.; Liu, X.; Fuller, C.J.; Susser, E.; Lu, J.; et al. The Psychological Impact of the SARS Epidemic on Hospital Employees in China: Exposure, Risk Perception, and Altruistic Acceptance of Risk. Can. J. Psychiatry 2009, 54, 302-311. [CrossRef] [PubMed]

14. García-Fernández, L.; Romero-Ferreiro, V.; López-Roldán, P.D.; Padilla, S.; Calero-Sierra, I.; Monzó-García, M.; Pérez-Martín, J.; Rodriguez-Jimenez, R. Mental health impact of COVID-19 pandemic on Spanish healthcare workers. Psychol. Med. 2020, 1-3. [CrossRef]

15. Collopy, K.T.; Kivlehan, S.M.; Snyder, S.R. Are you under stress in EMS. Understanding the slippery slope of burnout and PTSD. EMS World 2012, 41, 47-50, 52-56. [PubMed]

16. Carmassi, C.; Foghi, C.; Dell'Oste, V.; Cordone, A.; Bertelloni, C.A.; Bui, E.; Dell'Osso, L. PTSD symptoms in healthcare workers facing the three coronavirus outbreaks: What can we expect after the COVID-19 pandemic. Psychiatry Res. 2020, 292, 113312. [CrossRef] [PubMed]

17. Einav, S.; Shalev, A.Y.; Ofek, H.; Freedman, S.; Matot, I.; Weiniger, C.F. Differences in psychological effects in hospital doctors with and without post-traumatic stress disorder. Br. J. Psychiatry 2008, 193, 165-166. [CrossRef]

18. De Pablo, G.S.; Vaquerizo-Serrano, J.; Catalan, A.; Arango, C.; Moreno, C.; Ferre, F.; Shin, J.I.; Sullivan, S.; Brondino, N.; Solmi, M.; et al. Impact of coronavirus syndromes on physical and mental health of health care workers: Systematic review and meta-analysis. J. Affect. Disord. 2020, 275, 48-57. [CrossRef]

19. Miguel-Puga, J.A.; Cooper-Bribiesca, D.; Avelar-Garnica, F.J.; Sanchez-Hurtado, L.A.; Colin-Martínez, T.; Espinosa-Poblano, E.; Anda-Garay, J.C.; González-Díaz, J.I.; Segura-Santos, O.B.; Vital-Arriaga, L.C.; et al. Burnout, depersonalization, and anxiety contribute to post-traumatic stress in frontline health workers at COVID-19 patient care, a follow-up study. Brain Behav. 2021, 11, e02007. [CrossRef]

20. Moreno-Jiménez, J.E.; Blanco-Donoso, L.M.; Chico-Fernández, M.; Hofheinz, S.B.; Moreno-Jiménez, B.; Garrosa, E. The Job Demands and Resources Related to COVID-19 in Predicting Emotional Exhaustion and Secondary Traumatic Stress among Health Professionals in Spain. Front. Psychol. 2021, 12, 564036. [CrossRef] [PubMed]

21. Kroenke, K.; Spitzer, R.L.; Williams, J.B.W. The PHQ-9: Validity of a brief depression severity measure. J. Gen. Intern. Med. 2001, 16, 606-613. [CrossRef] [PubMed] 
22. Mazzotti, E.; Fassone, A.; Picardi, E.; Sagoni, L.; Ramieri, I.; Lega, D.; Camaioni, D.; Abeni, P.P. Il Patient Health Questionnaire (PHQ) per lo Screening dei Disturbi Psichiatrici: Uno Studio di Validazione nei Confronti Della Intervista Clinica Strutturata per il DSM-IV Asse I (SCID-I). The Patient Health Questionnaire (PHQ) for the Screening of Psychiatric Disorders: A Validation Study Versus the Structured Clinical Interview for DSM-IV axis I (SCID-I). J. Psychopathol. 2003, 9, 235-242. Available online: https:/ / www.jpsychopathol.it/article/il-patient-health-questionnaire-phq-per-lo-screening-dei-disturbi-psichiatrici-unostudio-di-validazione-nei-confronti-della-intervista-clinica-strutturata-per-il-dsm-iv-asse-i-scid-i/ (accessed on 10 June 2020).

23. Spitzer, R.L.; Kroenke, K.; Williams, J.B.W.; Löwe, B. A Brief Measure for Assessing Generalized Anxiety Disorder: The GAD-7. Arch. Intern. Med. 2006, 166, 1092-1097. [CrossRef] [PubMed]

24. Bastien, C.H.; Vallières, A.; Morin, C.M. Validation of the Insomnia Severity Index as an outcome measure for insomnia research. Sleep Med. 2001, 2, 297-307. [CrossRef]

25. Castronovo, V.; Galbiati, A.; Marelli, S.; Brombin, C.; Cugnata, F.; Giarolli, L.; Anelli, M.M.; Rinaldi, F.; Ferini-Strambi, L. Validation study of the Italian version of the Insomnia Severity Index (ISI). Neurol. Sci. 2016, 37, 1517-1524. [CrossRef] [PubMed]

26. Christianson, S.; Marren, J. The Impact of Event Scale-Revised (IES-R). Med. Surg. Nurs. 2012, 21, 321-323.

27. Craparo, G.; Faraci, P.; Rotondo, G.; Gori, A. The Impact of Event Scale-Revised: Psychometric properties of the Italian version in a sample of flood victims. Neuropsychiatr. Dis. Treat. 2013, 9, 1427-1432. [CrossRef] [PubMed]

28. Creamer, M.; Bell, R.; Failla, S. Psychometric properties of the Impact of Event Scale-Revised. Behav. Res. Ther. 2003, 41, 1489-1496. [CrossRef] [PubMed]

29. Maslach, C.; Jackson, S.E. The measurement of experienced burnout. J. Organ. Behav. 1981, 2, 99-113. [CrossRef]

30. Sirigatti, S.; Stefanile, C.; Menoni, E. Per un Adattamento Italiano del Maslach Burnout Inventory (MBI) [Toward an Italian Version of the Maslach Burnout Inventory (MBI)]. Boll. Psicol. Appl. 1988, 187-188, 33-39. Available online: https://www.researchgate.net/publication/233992983_Per_un_adattamento_italiano_del_Maslach_Burnout_Inventory_MBI_ Toward_an_Italian_version_of_the_Maslach_Burnout_Inventory_MBI (accessed on 24 March 2020).

31. Wheeler, D.; Vassar, M.; Worley, D.A.; Barnes, L.L.B. A Reliability Generalization Meta-Analysis of Co-efficient Alpha for the Maslach Burnout Inventory. Educ. Psychol. Meas. 2011, 71, 231-244. [CrossRef]

32. Perrin, P.C.; McCabe, O.L.; Everly, G.S.; Links, J.M. Preparing for an Influenza Pandemic: Mental Health Considerations. Prehospital Disaster Med. 2009, 24, 223-230. [CrossRef] [PubMed]

33. Gębska, M.; Kołodziej, Ł.; Dalewski, B.; Pałka, Ł.; Sobolewska, E. The Influence of the COVID-19 Pandemic on the Stress Levels and Occurrence of Stomatoghnatic System Disorders (SSDs) among Physiotherapy Students in Poland. J. Clin. Med. 2021, 10, 3872. [CrossRef] [PubMed]

34. Preti, E.; Di Mattei, V.; Perego, G.; Ferrari, F.; Mazzetti, M.; Taranto, P.; Di Pierro, R.; Madeddu, F.; Calati, R. The Psychological Impact of Epidemic and Pandemic Outbreaks on Healthcare Workers: Rapid Review of the Evidence. Curr. Psychiatry Rep. 2020, 22, 43. [CrossRef] [PubMed]

35. Deng, J.; Zhou, F.; Hou, W.; Silver, Z.; Wong, C.Y.; Chang, O.; Huang, E.; Zuo, Q.K. The prevalence of depression, anxiety, and sleep disturbances in COVID-19 patients: A meta-analysis. Ann. N. Y. Acad. Sci. 2020, 1486, 90-111. [CrossRef] [PubMed]

36. Bassi, M.; Negri, L.; Delle Fave, A.; Accardi, R. The relationship between post-traumatic stress and positive mental health symptoms among health workers during COVID-19 pandemic in Lombardy, Italy. J. Affect. Disord. 2020, 280 Pt B, 1-6. [CrossRef]

37. Dalewski, B.; Palka, L.; Kiczmer, P.; Sobolewska, E. The Impact of SARS-CoV-2 Outbreak on the Polish Dental Community's Standards of Care-A Six-Month Retrospective Survey-Based Study. Int. J. Environ. Res. Public Health 2021, 18, 1281. [CrossRef]

38. Conti, C.; Fontanesi, L.; Lanzara, R.; Rosa, I.; Porcelli, P. Fragile heroes. The psychological impact of the COVID-19 pandemic on health-care workers in Italy. PLoS ONE 2020, 15, e0242538. [CrossRef] [PubMed]

39. Rossi, R.; Socci, V.; Pacitti, F.; Di Lorenzo, G.; Di Marco, A.; Siracusano, A.; Rossi, A. Mental Health Outcomes among Frontline and Second-Line Health Care Workers during the Coronavirus Disease 2019 (COVID-19) Pandemic in Italy. JAMA Netw. Open 2020, 3, e2010185. [CrossRef] [PubMed]

40. Sanghera, J.; Pattani, N.; Hashmi, Y.; Varley, K.F.; Cheruvu, M.S.; Bradley, A.; Burke, J.R. The impact of SARS-CoV-2 on the mental health of healthcare workers in a hospital setting-A Systematic Review. J. Occup. Health 2020, 62, e12175. [CrossRef] [PubMed]

41. Serrano-Ripoll, M.J.; Meneses-Echavez, J.F.; Ricci-Cabello, I.; Fraile-Navarro, D.; Fiol-Deroque, A.M.; Pastor-Moreno, G.; Castro, A.; Ruiz-Pérez, I.; Campos, R.Z.; Gonçalves-Bradley, D.C. Impact of viral epidemic outbreaks on mental health of healthcare workers: A rapid systematic review and meta-analysis. J. Affect. Disord. 2020, 277, 347-357. [CrossRef] [PubMed]

42. Barello, S.; Palamenghi, L.; Graffigna, G. Stressors and Resources for Healthcare Professionals during the COVID-19 Pandemic: Lesson Learned From Italy. Front. Psychol. 2020, 11, 2179. [CrossRef] [PubMed]

43. Barello, S.; Palamenghi, L.; Graffigna, G. Burnout and somatic symptoms among frontline healthcare professionals at the peak of the Italian COVID-19 pandemic. Psychiatry Res. 2020, 290, 113129. [CrossRef] [PubMed]

44. Maslach, C.; Leiter, M.P. Understanding the burnout experience: Recent research and its implications for psychiatry. World Psychiatry 2016, 15, 103-111. [CrossRef]

45. Shah, K.; Kamrai, D.; Mekala, H.; Mann, B.; Desai, K.; Patel, R.S. Focus on Mental Health During the Coronavirus (COVID-19) Pandemic: Applying Learnings from the Past Outbreaks. Cureus 2020, 12, e7405. [CrossRef] [PubMed]

46. Shah, K.; Chaudhari, G.; Kamrai, D.; Lail, A.; Patel, R.S. How Essential Is to Focus on Physician's Health and Burnout in Coronavirus (COVID-19) Pandemic? Cureus 2020, 12, e7538. [CrossRef] 
47. Panagioti, M.; Geraghty, K.; Johnson, J.; Zhou, A.; Panagopoulou, E.; Chew-Graham, C.; Peters, D.; Hodkinson, A.; Riley, R.; Esmail, A. Association Between Physician Burnout and Patient Safety, Professionalism, and Patient Satisfaction: A Systematic Review and Meta-analysis. JAMA Intern. Med. 2018, 178, 1317-1330. [CrossRef]

48. Yang, L.; Yin, J.; Wang, D.; Rahman, A.; Li, X. Urgent need to develop evidence-based self-help interventions for mental health of healthcare workers in COVID-19 pandemic. Psychol. Med. 2021, 51, 1775-1776. [CrossRef]

49. Chong, M.-Y.; Wang, W.-C.; Hsieh, W.-C.; Lee, C.-Y.; Chiu, N.-M.; Yeh, W.-C.; Huang, O.-L.; Wen, J.-K.; Chen, C.-L. Psychological impact of severe acute respiratory syndrome on health workers in a tertiary hospital. Br. J. Psychiatry 2004, 185, 127-133. [CrossRef] [PubMed]

50. Buselli, R.; Baldanzi, S.; Corsi, M.; Chiumiento, M.; Del Lupo, E.; Carmassi, C.; Dell'Osso, L.; Cristaudo, A. Psychological Care of Health Workers during the COVID-19 Outbreak in Italy: Preliminary Report of an Occupational Health Department (AOUP) Responsible for Monitoring Hospital Staff Condition. Sustainability 2020, 12, 5039. [CrossRef]

51. Søvold, L.E.; Naslund, J.A.; Kousoulis, A.A.; Saxena, S.; Qoronfleh, M.W.; Grobler, C.; Münter, L. Prioritizing the Mental Health and Well-Being of Healthcare Workers: An Urgent Global Public Health Priority. Front. Public Health 2021, 9, 679397. [CrossRef] [PubMed]

52. Tomlin, J.; Dalgleish-Warburton, B.; Lamph, G. Psychosocial Support for Healthcare Workers during the COVID-19 Pandemic. Front. Psychol. 2020, 11, 1960. [CrossRef] [PubMed]

53. Maulik, P.K.; Thornicroft, G.; Saxena, S. Roadmap to strengthen global mental health systems to tackle the impact of the COVID-19 pandemic. Int. J. Ment. Health Syst. 2020, 14, 57. [CrossRef] [PubMed]

54. Franklin, P.; Gkiouleka, A. A Scoping Review of Psychosocial Risks to Health Workers during the COVID-19 Pandemic. Int. J. Environ. Res. Public Health 2021, 18, 2453. [CrossRef] [PubMed] 